\title{
Drug Induced Hepatotoxicity - An Ongoing Challenge
}

\author{
Mehr Fatima, Syed Ijaz Hussain Zaidi \\ - - - - - - - - - - - - - - - - - - - - - - - - - - -
}

ABSTRACT:

Drug induced liver injury is one of the main factor of liver failure and acute liver damage world wide with high incidence in western countries. Liver injury can be intrinsic (dose dependant) or idiosyncratic (dose independent). However idiosyncratic type is considered to be mainly responsible for drug induced liver damage. Binding of reactive metabolites of drugs to tissue proteins and oxidative stress is the possible cellular mechanism involved in this process. Moreover, some antibiotics, anti-epileptics, nonsteroidal anti-inflammatory drugs etc are more likely to induce liver damage in high risks groups that includes females, elderly and obese people. HLA halotype and variation in protein expression also plays an important role in this context. Various studies are available regarding clinical features, histopathological features, diagnosis and management related to antibiotics and acetaminophen induced liver damage. $\mathrm{N}$ acetylcysteine is commonly available antidote for drug induced hepatic damage. Role of other pharmacological agents as an antidote requires further studies. However, liver transplantation should be considered with drug induced lethal liver failure.
\end{abstract}

Key Words: Liver injury, drug induced liver damage, antibiotic, liver histopathology, liver enzymes, antidote.

How to cite this Article:

Fatima M, Zaidi SIH. Drug Induced Hepatotoxicity - An Ongoing Challenge. J Bahria Uni Med Dental Coll. 2020;10(3): 244-8 DOI: https://doi.org/10.51985/JBUMDC2020007

\section{INTRODUCTION:}

Drug induced liver injury can be stated as the damage to liver tissue caused by several medicines, plants and exogenous chemicals excluding other etiological factors which affects liver enzymes and liver function. In USA, drug induced liver damage is the main contributory factor to acute hepatic damage and it accounts for $13 \%$ of acute hepatic failure cases. $^{1}$

Liver is the main organ involved in detoxification of drugs. Liver health can be affected by drugs either by intrinsic (dose dependent) or idiosyncratic (dose independent manner). Some drugs like Acetaminophen can induce damage if given in excessive dosages. Idiosyncratic drug induced liver injury is thought to be unpredictable and accounts for most cases of drug induced liver injury. Patients with underlying liver diseases can have the possibility of having idiosyncratic drug induced liver damage. Data regarding drug induced liver injury cases have been found in Western as well as Asian countries. It is reported to have the annual incidence of 13.5 cases per 100,000 in France, 12 cases per 100,000 in Korea and 19.1cases per 100,000 in Iceland. ${ }^{2,3}$ Considering that information on drug induced liver injury is not available in the form of a database or national registry in Pakistan, a retrospective study conducted at a major tertiary care hospital

「-ー - - - - - - - - - -

I Mehr Fatima (M.Phil Postgraduate Student)

Senior Lecturer, Department of Pharmacology

Bahria University Medical and Dental College, Karachi

I Email: mehr_fatima@yahoo.com

I

Syed Ijaz Hussain Zaidi

Associate Professor, Department of Pharmacology

I Bahria University Medical and Dental College, Karachi

I Received: 27-Jan-2020

I Accepted: 17-Jun-2020

L - - - - - - - - - - - - - - I in Pakistan identified $31.1 \%$ cases of drug induced liver injury on the basis of Roussel Uclaf Causality Assessment Method (RUCAM), over a 7 year period. ${ }^{4}$ Many drugs have been withdrawn from the market due to this reason. Since drug induced liver injury is common reason of liver failure in USA and Europe, practice guidelines have been set up by American college of Gastroenterology regarding diagnosis and management of idiosyncratic drug induced liver injury. ${ }^{2,3}$

Earlier studies showed that the drugs were either removed from the market such as Ximelagalran, Troglitazone, Bromlenac, Benoxaprofen or received black box warning such as Valproic acid, Ketoconazole, Isoniazid, Rifampin, Nicotinic acid, Dantrolene, Telethromycin, Infliximab, Nefazadone due to their hepatotoxic effects that might be dose dependant. ${ }^{5}$

Drug induced hepatotoxicity is an ongoing challenge that needs specific addressing particularly in our part of world as available literature is scanty.

\section{METHODOLOGY:}

Different search engines like Google scholar, Pubmed and Cochrane library were used for writing this review. Around 69 articles were found for the year 2008-2019. From these 16 were of paediatrics, 6 were animal studies, 4 were abstract only, 2 were in non English version and therefore were excluded. A total of 41 articles are used in the write up of this review focusing on drugs that commonly caused hepatotoxicity in adults and obese individuals. The key words used were liver injury, liver enzymes, drug induced liver injury, antidote, liver histopathology and antibiotics and liver injury. 


FLOW CHART
$\begin{gathered}\text { Literature Search Period: 2008-2019 } \\ \text { Studies Searched from 2008-2019=69 } \\ \text { Total Studies Excluded = 28 (Paediatrics }=16, \\ \text { Animal Studies }=6, \text { Abstract only=4, Non- } \\ \text { English Version=2) }\end{gathered}$
Total Articles covered in this Review = 41

\section{LITERATURE REVIEW:}

\section{Risk Factors For Drug Induced Liver Injury:}

Females and elderly people, patients with chronic liver disease and HIV infection are more prone to be affected by drug induced liver injury. However, data available regarding these factors is insufficient. Females are more vulnerable to the effects of some drugs. Drug induced liver damage in females have been linked to some specific drugs like Nitrofurantoin, Azithromycin, Erythromycin, Flucoloxacillin, Amoxicillin, Fluoroquinolones, Minocycline and Isoniazid. Different age groups are being affected by different drugs. Isoniazid is more likely to cause liver damage in old aged patients whereas Aspirin and Valproate is a risk factor for younger age group. Since processes of pharmacokinetics including absorption, metabolism and elimination varies, in elderly might be the factor of drug induced liver injury. A prospective study conducted on Spanish population also reported increased risk of drug induced liver damage in older women which might be due to loss of cell repair and regeneration processes as the result of aging. Patients with underlying liver disease as a risk factor is arguable but patients with Hepatitis B, Hepatitis C taking anti-tubercular and antiretroviral drugs are at risk. ${ }^{6,7}$ Alcohol intake and diabetes is another risk factor for severity of drug induced liver injury. ${ }^{9}$

Risk of drug induced liver injury is also high in obese people. Drugs like Corticosteroid, Methotrexate, Tamoxifen, Tetracycline and Nucleotide reverse transcriptase inhibitors may worsen non alcoholic fatty liver disease in these individuals. Drugs like Losartan, Ticlopidine, Acetaminophen, Omeprazole, Halothane and Isoflurane are more likely to induce acute liver damage in obese individuals. ${ }^{10,11}$

\section{Mechanism of Drug induced liver injury:}

Intrinsic type of drug induced liver damage occur in acetaminophen toxicity. In this type, liver is damaged is by the production of large amount of reactive metabolite which exceeds liver capacity to convert it into nontoxic compounds as well as it depletes Glutathione levels which finally leads to hepatotoxicity. ${ }^{12,13}$

Mechanism of liver injury by idiosyncratic type can be classified into two types; Hypersensitive- type reactions (immunologic) and Metabolic type of injuries. ${ }^{12,13}$
Hypersensitivity type of drug induced liver injury is reported to occur in 23 to $37 \%$ of all idiosyncratic reactions. It is produced due to covalent binding of reactive metabolites of drugs to tissue proteins which provoke immune mediated reaction and direct hepatic damage. Metabolic mechanism mainly involves mitochondrial injury and oxidative stress. Liver damage can occur as a consequence of oxidative stress. Since reactive oxygen specie damages nucleus and main enzymes, an immune mediated liver injury may happen. This can be a result of imbalance between free oxygen radical formation and cellular defense mechanism. ${ }^{12,13}$

Enzymes of phase 1 and 2 expression and cytokine expression are also linked to hepatotoxicity induced by drugs. Formation of toxic metabolites of anti tubercular drugs is related to CYP2E1 enzyme, while toxic metabolite of Diclofenac is CYP2C8 enzyme. It is also suggested that there is an association between antibiotic induced hepatotoxicity and HLA halotypes. Studies on genome showed that Amoxicillin is related to 10 times increase in risk of developing hepatotoxicity due to HLA halotypes HLA-DRB1 *1501-DRB5, *0101-DQB1 *0602. Not only this, variation in protein expression of different drug transporters has been reported to contribute in hepatotoxicity induced by drugs. In this context, multi drug resistant protein and bile salt export pump have been associated with drug induced hepatotoxicity. ${ }^{14}$

\section{1) Antibiotic induced hepatotoxicity:}

Studies conducted in western countries have shown Amoxicillin as the common antibiotic affecting liver conditions leading to hepatic damage. ${ }^{15}$

In a study conducted in Iceland for the period of 2 years ,Amoxicillin was determined to be one of the most common drug causing liver injury. A study was conducted on 35252 patient in Iceland. 1 out of 2350 outpatients had liver injury whereas risk of developing liver injury was higher in inpatients. 1 out of 729 inpatients treated with Amoxicillin also developed liver damage. There was no obvious reason for this variation in incidence of inpatient versus outpatient amoxicillin treated groups. However limited data regarding route of drug administration is available. It was reported that Amoxicillin-clavulanate caused delayed and late type of liver injury with mean latency of 16 and 30 days from the beginning of the therapy. ${ }^{16}$

Although clinical feature of hepatotoxicity caused by Amoxicillin varies but jaundice was the most observed symptom. Other predominant symptoms were tiredness, anorexia and itching. In a large cohort study $(n=1038)$ conducted in Spain, with 11\% representing Amoxicillin induced hepatic injury being the main cause of idiosyncratic drug induced liver injury. Liver enzyme (ALT and AST) levels reached at the highest level after 4-5 days where as ALP and total bilirubin at the highest level at around 17 and 12 days. Cholestatic features were more marked on liver 
biopsies. 2 participants were reported to have asymptomatic elevation of liver enzymes. Very few subjects were in need of liver transplantation. ${ }^{17}$ Hepatocellular hepatic granuloma were caused by amoxicillin induced hepatic injury. ${ }^{18}$

\section{2) Azithromycin:}

Azithromycin belongs to macrolide class of antibiotic. It is used for the treatment of mild to moderate sinopulmonary diseases. It causes idiosyncratic hepatotoxicity with variable clinical presentation. In a prospective cohort study, the most common symptoms were jaundice, abdominal pain ,nausea and pruritis. In a study, Azithromycin was given for 4 days (mean duration). Liver enzymes levels were found to be deranged 14 days after the cessation of azithromycin . Liver biopsy showed hepatocellular liver injury (56\%), cholestatic $(33 \%)$ and mixed pattern $(11 \%)$. Antinuclear antibodies were more likely to be present in patients with hepatocellular injury than with cholestatic or mixed type. ${ }^{19}$

\section{3) Fluoroquinolones:}

Fluoroquinolnes are a widely used antibiotic due to better coverage against microbes and good efficacy. Previous studies reported that they cause idiosyncratic drug injury. Common clinical features with liver injury caused by fluoroquinolones were rash, fever and increased eosinophilia. Fluoroquinolones caused liver damage with sudden onset. It had short latency period. Liver biopsy showed hepatocellular, cholestatic and mixed type of liver injury. Corticosteriods were effective for patients with features of hypersensitivity. Patients with Fluoroquinolones induced liver damage rarely developed acute and chronic liver failure. Moxifloxacin, Levofloxacin and Gatifloxacin causes acute liver failure with the incidence of $(2.1,6.1,6.6$ per 10 million prescriptions), this is less than Amoxicillin - clavulanate(10 in 1 million prescription). ${ }^{20}$

\section{4) Nitrofurantoin:}

A prospective cohort study reported that Nitrofurantoin induces liver injury with particular autoimmune features with female preponderance. Studies indicated that time from initiation of therapy to the identification of deranged liver enzyme level that is latency period in Nitrofurantoin induced liver damage can be quite prolonged. It differs from 4 days to more than 5 years. Liver injury patterns were mainly hepatocellular. ${ }^{21,22}$

\section{5) Isoniazid:}

Tuberculosis is a chronic infectious disease of respiratory system affecting 9 million people world wide. Isoniazid is the drug of choice for latent tuberculosis. Other most commonly used first line anti tubercular drugs Rifampicin and Pyrazanamide can also induce liver damage. Researchers revealed that, in most cases, no symptoms can be produced in case of liver injury caused by isoniazid. It can be identified by measuring serum liver enzyme levels. However , previous studies showed that the main features of liver injury caused by Isoniazid were nausea, vomiting, jaundice, fever, eosinophilia, malaise and fatigue. Changes in liver enzyme levels were detected as early as 1 week and as late as 9 months. Pattern of liver injury was hepatocellular with multilobular necrosis and mononuclear infiltrate which was difficult to differentiate from viral hepatitis. In case of long term treatment, isoniazid produced lupus like autoimmune reaction with raised antinuclear antibodies. Few studies found out the association between chronic hepatitis B virus and anti-tubercular induced liver injury. Hepatitis B virus carrier had more critical liver damage with anti-tubercular drugs as compared to non-carriers. ${ }^{23,24}$

Since isoniazid is mainly metabolized by enzyme Nacetyltranferase, slow acetylators are at higher risk of having isoniazid induced injury. Based on the expression of halotypes, patients were categorized as rapid, intermediate and slow acetylors as reported by earlier studies. Slow acetylators were deprived of the NAT $2 * 4$ allele or other rapid NAT2 alleles whereas rapid acetylators had homozygotes NAT2 $* 4$ and intermediate acetylators possessed the NAT2 $* 4 / * 5 \mathrm{~B}, * 4 / * 6 \mathrm{~A}$, or $* 4 / * 7 \mathrm{~B}$ halotypes. NAT2 $* 4$, $* 6$ and $* 7$ alleles were present in many Asian populations. NAT2 $* 7$ were peculiar allele in Asians, while NAT2*5 was commonly found in Caucasians and Africans. ${ }^{25}$

\section{6) Acetaminophen induced hepatotoxicity:}

Acetaminophen is widely used analgesic and is one of the main cause of acute liver failure. It is commonly available over the counter drug. Almost 30,000 people are hospitalized annually in USA for treatment of paracetamol intoxication. Paracetamol induced liver toxicity is mainly responsible for almost $48 \%$ of liver failure diagnosis. It occurs due to formation of large amount of reactive metabolite $\mathrm{N}$ acetyl $\mathrm{p}$ benzoquinone (NAPQI). This depletes Glutathione level and its capacity to convert it to non toxic metabolite. ${ }^{26,27}$

Symptoms of acute liver failure were anorexia, abdominal discomfort, jaundice and fever progressing to hepatic encepaholopathy 0-7 days after the onset of jaundice in case of acetaminophen toxicity. ${ }^{27}$ Studies have revealed that patients may present with the specific signs of decreased level of consciousness, increased serum level of acetaminophen and lactic acidosis several hours after acetaminophen intake. NAPQI disrupts mitochondrial function which can cause early lactic acidosis. ${ }^{27,28}$

A review study has reported differences in metabolic enzyme level due to genetic variability as the risk factor for paracetamol intoxication. Randomized control trial of 145 patients reported that there was an association in Alanine aminotranferase level at the dose of $4 \mathrm{~g}$ paracetamol administered for 4 days. ${ }^{29}$

Another review article has reported common hepatic damage caused by drugs. Isoniazid, disulfiram and acetaminophen causes acute hepatic necrosis. Nitrofurantoin and Minocycline causes drug induced autoimmune hepatitis. ${ }^{30}$ 


\section{DIAGNOSIS:}

Diagnosis is mainly based on complete history ,patient age and sex, history of drug reactions, history of liver disease and drug abuse, main disease for which the drug was prescribed, drug exposure, other medications if used for 3 months before the onset of liver injury. Signs and symptoms include weakness, abdominal pain, dark urine, presence and absence of jaundice, fever, liver tenderness etc. Abnormal liver biochemistry (Biliribin, ALT, ALP, INR, PT), laboratory test to be done to exclude other causes (IgM anti- HAV, $\mathrm{IgM}$ anti $\mathrm{HBc}, \mathrm{HBs}$ antigen, anti- HCV. Antinuclear antibodies, and liver imaging (abdominal ultrasound, CT and MRI) are used to diagnose liver damage, however liver biopsy is not necessary. ${ }^{31,32,33}$

Currently many new serological, biochemical and histological biomarkers are used to detect drug induced liver damage. These included apoptosis- cytokeratin-18 fragments (CK18Fr), soluble Fas and Fas ligand (sFas/sFasL), soluble TNF-á and TNF receptor related (sTNF-á/ sTNFR), and soluble TNF-related apoptosis-inducing ligand, cell necrosisrelated full-length CK18, high-mobility group protein B1, and micro RNA, specific mitochondrial biomarkers, circulating autoantibodies targeting drug-metabolizing enzymes such as cytochrome $\mathrm{P} 450$, biomarkers indicating cholestasis, as well as genetic biomarkers to drug induced liver damage, such as the genetic polymorphisms of HLA, drug metabolizing enzymes and drug transport proteins. However, these markers showed poor specificities for the diagnosis of drug induced liver injury, and their value for clinical use still needs to be further tested. Moreover only acetaminophen protein adduct was a peculiar biomarker of acetaminophen related liver injury. ${ }^{34}$

\section{Management of Drug Induced Liver Injury:}

Following are main principles of management for drug induced liver injury;

1) Withdrawal of drug immediately if it was not the critical drug used for the treatment of any specific disease. Timely withdrawal of the drug affecting liver is important. It was estimated that $95 \%$ of the patient ,responded to drug withdrawal. ${ }^{34,35}$

2) Based on clinical signs and symptoms use appropriate anti-inflammatory and hepatoprotective agents to treat drug induced liver injury. ${ }^{34}$

3) Corticosteroids had also been used to treat drug induced liver injury. But so far no controlled trial has been reported. ${ }^{35,37}$

4) FDA has recommended $\mathrm{N}$-acetylcysteine as the anti dote for all types of drug induced liver injury in 2004. It should be administered at the dose of $(50-150 \mathrm{mg} / \mathrm{kg})$ for 3 days. Previous studies reported its role in early stage of liver failure. However further investigations are required to determine its role in moderate and severe liver damage. In case of acetaminophen induced liver damage, use of $\mathrm{N}$ -acetylcysteine within 24 hours of acetaminophen ingestion is found to be effective. ${ }^{35,36,37}$

5) Liver transplantation is required for those cases of drug induced acute liver failure who presented with decompensated cirrhosis, hepatic encephalopathy or coagulation disorders. ${ }^{34}$

6)In an immune mediated liver injury, role of glucocorticoid and uricosuric agents were not yet clear. More over, in patients with no improvement after drug withdrawal ,corticosteroids may be effective with allergic features of drug induced liver injury. ${ }^{38}$

7)In case of non acetaminophen induced liver injury, use of $\mathrm{N}$ acetylcysteine is restricted. Molecular adsorbent recirculating system and plasma exchange in management of drug induced liver injury has demonstrated its effectiveness but it still requires further work up. ${ }^{39}$

8)The active ingredient of milk of thistle, Silmyrin has shown protective effects in such patients. ${ }^{40}$

So far, few preclinical studies have also revealed the role of some medicinal herbs and food in preventing drug induced liver injury which is an open avenue for future. ${ }^{41}$

\section{CONCLUSION:}

Since drug use can lead to hazardous and troublesome effects for body tissues, especially liver which can be fatal, therefore, drug use should be rationalized in order to prevent drug induced liver damage. Moreover, early diagnosis and management of individuals who are either at high risk or exposed to drugs, might be helpful in preventing serious consequences of liver damage. Furthermore, studies regarding drug induced liver damage in our setup are lacking and more research work should be carried out in order to explore role of drugs and their antidotes. Thus, the matter of drug induced hepatotoxicity is an on-going challenge for health professionals at preventive, diagnostic and management horizons.

Author Contribution:
Mehr Fatima: Selection of topic, introduction \& literature |
review
Syed Ijaz Hussain Zaidi: Abstract writing, conclusion and
methodology

\section{REFERENCES}

1) Suk KT, Kim DJ. Drug-induced liver injury: present and future. Clinical and molecular hepatology. 2012 ; 18(3):24957.

2) Licata A. Adverse drug reactions and organ damage: the liver. European journal of internal medicine. 2016;(28):9-16.

3) Kim SH, Naisbitt DJ. Update on advances in research on idiosyncratic drug-induced liver injury. Allergy, asthma \& immunology research. $2016 ; 1 ; 8$ (1):3-11.

4) Abid A, Subhani F, Kayani F, Awan S, Abid S. Drug induced liver injury is associated with high mortality-A study from a tertiary care hospital in Pakistan. Plos one. 2020; 15(4): $\mathrm{e} 0231398$. 
5) Lammert C, Einarsson S, Saha C, Niklasson A, Bjornsson E, Chalasani N. Relationship between daily dose of oral medications and idiosyncratic drug-induced liver injury: search for signals. Hepatology. 2008 ;47 (6):2003-9.

6) Leise MD, Poterucha JJ, Talwalkar JA. Drug-induced liver injury. InMayo clinic proceedings 2014;89(1): 95-106. Elsevier.

7) Medina-Caliz I, Robles-Diaz M, Garcia-Muñoz B, Stephens C, Ortega-Alonso A, Garcia-Cortes M, et al. Definition and risk factors for chronicity following acute idiosyncratic druginduced liver injury. Journal of hepatology. 2016 ; 1;65(3):53242.

8) Chalasani N, Björnsson E. Risk factors for idiosyncratic druginduced liver injury. Gastroenterology. 2010 ; 138(7):224659.

9) Ghabril M, Chalasani N, Björnsson E. Drug-induced liver injury: a clinical update. Current opinion in gastroenterology. $2010 ; 26(3): 222-6$

10) Fromenty B. Drug-induced liver injury in obesity. Journal of hepatology. $2013 ; 1 ; 58(4): 824-6$.

11) Leung TM, Nieto N. CYP2E1 and oxidant stress in alcoholic and non-alcoholic fatty liver disease. Journal of hepatology. 2013; ;58(2):395-8.

12) Alempijevic T, Zec S, Milosavljevic T. Drug-induced liver injury: Do we know everything?. World journal of hepatology. 2017;9(10):491-502

13) Chen M, Suzuki A, Borlak J, Andrade RJ, Lucena MI. Druginduced liver injury: Interactions between drug properties and host factors. Journal of hepatology. 2015 ; 63(2):503-14.

14) Njoku D. Drug-induced hepatotoxicity: metabolic, genetic and immunological basis. International journal of molecular sciences. 2014 ;15(4):6990-7003.

15) Björnsson ES. Incidence and outcomes of DILI in Western patients. Clinical liver disease. 2014 ; 4(1):9-11.

16) Björnsson ES. Drug-induced liver injury due to antibiotics. Scandinavian journal of gastroenterology. 2017; 52(6-7):61723.

17) Delemos AS, Ghabril M, Rockey DC, Gu J, Barnhart HX, Fontana RJ, et al. Amoxicillin-clavulanate-induced liver injury. Digestive diseases and sciences. 2016 ; 61(8):240616.

18) Robles M, Toscano E, Cotta J, Isabel Lucena M, J Andrade R. Antibiotic-induced liver toxicity: mechanisms, clinical features and causality assessment. Current drug safety. 2010; 5(3):212-22.

19) Martinez MA, Vuppalanchi R, Fontana RJ, Stolz A, Kleiner DE. Clinical and histologic features of azithromycin-induced liver injury. Clinical gastroenterology and hepatology. 2015;13(2):369-76.

20) Fisher K, Vuppalanchi R, Saxena R. Drug-induced liver injury. Archives of Pathology and Laboratory Medicine. 2015 ;139(7):876-87.

21) de Boer YS, Kosinski AS, Urban TJ, Zhao Z, Long N. Features of autoimmune hepatitis in patients with drug-induced liver injury. Clinical Gastroenterology and Hepatology. 2017;15(1):103-12.

22) Chalasani N, Bonkovsky HL, Fontana R, Lee W, Stolz A. Features and outcomes of 899 patients with drug-induced liver injury: the DILIN prospective study. Gastroenterology. 2015;148(7):1340-52.

23) Metushi I, Uetrecht J, Phillips E. Mechanism of isoniazidinduced hepatotoxicity: then and now. British journal of clinical pharmacology. 2016;81(6):1030-6.
24) Wang NT, Huang YS, Lin MH, Huang B, Perng CL. Chronic hepatitis B infection and risk of antituberculosis drug-induced liver injury: Systematic review and meta-analysis. Journal of the Chinese Medical Association. 2016 ; 79(7):368-74.

25) Wattanapokayakit S, Mushiroda T, Yanai H, Wichukchinda N, Chuchottawon C. NAT2 slow acetylator associated with anti-tuberculosis drug-induced liver injury in Thai patients. The International Journal of Tuberculosis and Lung Disease. 2016; 20(10):1364-9.

26) Yoon E, Babar A, Choudhary M, Kutner M, Pyrsopoulos N. Acetaminophen-induced hepatotoxicity: a comprehensive update. Journal of clinical and translational hepatology. 2016 ;4(2):131-42.

27) Lancaster EM, Hiatt JR, Zarrinpar A. Acetaminophen hepatotoxicity: an updated review. Archives of toxicology. $2015 ; 89(2): 193-9$.

28) Eikemans BJ, Mauritz R, van Westerloo DJ. Early lactic acidosis after acetaminophen overdose. Netherlands Journal of Critical Care. 2016 ; 24(5):21-3.

29) Caparrotta TM, Antoine DJ, Dear JW. Are some people at increased risk of paracetamol-induced liver injury? A critical review of the literature. European journal of clinical pharmacology. 2018 ; 74(2):147-60.

30) Björnsson E. Drug-induced liver injury in clinical practice. Alimentary pharmacology \& therapeutics. 2010 ; 32(1):3-13.

31) Lopez AM, Hendrickson RG. Toxin-induced hepatic injury. Emergency Medicine Clinics. 2014 ;32(1):103-25

32) Hayashi PH, Fontana RJ. Clinical features, diagnosis, and natural history of drug-induced liver injury. In Seminars in liver disease 2014; 34(02):134-44

33) Agarwal VK, McHutchison JG, Hoofnagle JH, Network DI. Important elements for the diagnosis of drug-induced liver injury. Clinical Gastroenterology and Hepatology. 2010; ;8(5):463-70.

34) Yu YC, Mao YM, Chen CW, Chen JJ, Chen J. CSH guidelines for the diagnosis and treatment of drug-induced liver injury. Hepatology international. 2017; 11(3):221-41.

35) Chalasani NP, Hayashi PH, Bonkovsky HL, Navarro VJ, Lee WM. ACG Clinical Guideline: the diagnosis and management of idiosyncratic drug-induced liver injury. The American journal of gastroenterology. 2014;109(7):950-66.

36) Singh S, Hynan LS, Lee WM. Acute Liver Failure Study G. Improvements in hepatic serological biomarkers are associated with clinical benefit of intravenous $\mathrm{N}$-acetylcysteine in early stage non-acetaminophen acute liver failure. Dig Dis Sci. 2013;58(5):1397-402.

37) Tujios SR, Lee WM. Acute liver failure induced by idiosyncratic reaction to drugs: challenges in diagnosis and therapy. Liver International. 2018 ; 38(1):6-14.

38) Verma S, Kaplowitz N. Diagnosis, management and prevention of drug-induced liver injury. Gut. 2009 ; 58(11): 1555-64.

39) Lewis JH. The art and science of diagnosing and managing drug-induced liver injury in 2015 and beyond. Clinical Gastroenterology and Hepatology. 2015 ; 13(12):2173-89.

40) Hassan A, Fontana RJ. The diagnosis and management of idiosyncratic drug-induced liver injury. Liver International. 2019 ; 39(1):31-41.

41) Meng X, Li Y, Li S, Gan RY, Li HB. Natural Products for Prevention and Treatment of Chemical-Induced Liver Injuries. Comprehensive Reviews in Food Science and Food Safety. 2018 ; 17(2): 472-95. 